\title{
Numerical Computation of 3-Dimensional Supersonic Flow Field over Seamless Missiles
}

\author{
Hussain H. Al-Kayiem ', Ahmed K. Hussein ${ }^{2}$, Jalal M. Jaleel ${ }^{3}$ and Salam H. Hussain ${ }^{2}$ \\ 'Mechanical Engineering Department, Universiti Teknologi Petronas, 31750 Tronoh, Perak, \\ Malaysia; hussain_kayiem@petronas.com.my \\ ${ }^{2}$ Mechanical Engineering Department, College of Engineering, Babylon University- Babylon City - Iraq; \\ ahmedkadhim74@yahoo.com \\ ${ }^{3}$ University of Technology-Technical Teaching Department, Baghdad City - Iraq.
}

\begin{abstract}
Investigation of the flow field surrounding supersonic non uniform flying body is a challenge to the aerodynamics researchers. Such investigation by the traditional experimental technique in the wind tunnel is a time consuming and costly. The alternative option is the CFD simulation. This work deals with prediction of the primitive variables of supersonic flow over a missile body, which has a complex profile. Finite difference computational fluid dynamic methods were adopted to solve the governing equations of supersonic, inviscid, compressible, and three-dimensional flow over a missile body with no canard. To deal with complex shape of missile, the "body fitted coordinate system" technique is considered to convert the generated grid from space physical domain to 3-D computational domain. Time-marching MacCormack's explicit technique is used to solve the set of the finite difference discretization equations. The analysis was carried out at 1.5 Mach number. The numerical procedure adopted for this application is found to be capable to capture the shock waves created over the missile body. The explicit technique required about 4000 time steps to achieve the converged solution. The velocity and temperature results showed a good agreement with previously published results. The same approach can be adopted to solve for different Mach numbers for the same missile shape.
\end{abstract}

Keywords: CFD, Compressible Fluid Flow, Euler Equation, Missiles Aerodynamics, Numerical Methods, Supersonic Flow

\section{Nomenclature}

\begin{tabular}{|c|c|c|c|}
\hline$\underline{\text { Symbole }}$ & Description & Symbole & Description \\
\hline$a$ & Speed of sound. & $J$ & Jacobian of coordinate's transformation. \\
\hline CFL & Courant FridrichLewysstability condition. & $M_{\infty}$ & Free stream Mach number \\
\hline $\mathrm{C}_{\zeta}, \mathrm{C}_{\eta}, \mathrm{C}_{\xi}$ & $\begin{array}{l}\text { Artificial viscosity coefficients in } \xi, \eta \text {, and } \zeta \text {, } \\
\text { directions }\end{array}$ & $p_{\infty}$ & $\begin{array}{l}\text { Free stream pressure. } \\
\text { Flux vector. }\end{array}$ \\
\hline$e$ & Specific internal energy per unit mass & Q & Vector of conserved variables in body \\
\hline$E_{\mathrm{t}}$ & Total energy per unit volume & $R$ & $\begin{array}{l}\text { fitted coordinates. } \\
\text { Universal gas constant }\end{array}$ \\
\hline$E, F, G$ & Column vector in Cartesian coordinates & $\operatorname{Re}$ & Reynolds number \\
\hline$E, \bar{F}, \bar{G}$ & Column vector in body fitted coordinates. & $S Q_{1}$ & Artificial viscosity term \\
\hline$L$ & Length of the missile & $T_{\infty}$ & Free stream temperature. \\
\hline
\end{tabular}

${ }^{*}$ Author for correspondence 


$$
\begin{array}{ll}
t & \text { Time } \\
u, v, w & \text { Velocity components in } x, y \text {, and } z \text { directions } \\
U, V, W & \begin{array}{l}
\text { Contravariant velocity components in } \xi, \eta \\
\text { and } \zeta \text { directions }
\end{array}
\end{array}
$$

\section{Greek Symbols}

$\Delta t \quad$ Time step

$\rho \quad$ Density

$\alpha \quad$ Angle of attack.

$\delta \quad$ Boundary layer thickness.

$\Delta x \Delta y \Delta z \quad$ Spatial steps in physical domain.

$\Delta \zeta \Delta \eta \Delta \xi \quad$ Spatial steps in computational domain.

\section{Subscript}

$\begin{array}{ll}I, j, k & \begin{array}{l}\text { Node symbols indicates position in } \mathrm{x}, \mathrm{y} \text { and } \mathrm{z} \\ \text { directions }\end{array} \\ \infty & \text { Conditions at free stream } \\ \mathrm{o} & \text { Stagnation (total) conditions. }\end{array}$

\section{Superscript}

n Time level $t$

$\mathrm{n}+1 \quad$ Time level $(t+\Delta t)$

\section{Introduction}

Prediction of flow field behavior is very important in the design of supersonic aircrafts and rockets. Not so long past time, the experimental measurements and the analytical methods were used to estimate the properties of supersonic flow over a limited number of shapes. From the mathematical point of view, the purpose of obtaining solutions to the governing equations of fluid mechanics represents one of the most problems in engineering. In most cases, the mathematical formulations of the basic laws of fluid mechanics are expressed as Partial Differential Equations (PDEs). Second-order partial differential equations appear frequently and, therefore, are of particular interest in fluid mechanics and heat transfer. Generally, the governing equations of fluid mechanics form a set of coupled, non-linear PDEs which must be solved in an irregular domain subject to various initial and boundary conditions. For supersonic, three-dimensional shaped missile, the analytical methods were failed due to the high non-linearity nature of the governing equations. To design an aircraft or missile, thousands of tests were drawn in a supersonic wind tunnel which requires a hard and expensive work and consumes considerable time domain. At the present time, design of new aircraft or missile needs-at least- ten-thousand to one -hundred thousand hours of wind tunnel time Jameson 1989. In contrast, a numerical prediction provides similar result with short-time and good accuracy. Computation and the computer program may be changed easily to deal with any other sort of supersonic missile. In the numerical solution, the complication of solving the differential equations are overcome by replacing them with differences, calculated from a finite number of values associated with the computational nodes, which are distributed on a suitable grid over the solution domain.

Early computational work was reported by Weaterill and Forsey ${ }^{2}$ where they have calculated the flow field around a complex aircraft configuration using multiblock grid approach coupled with explicit finite volume Euler algorithm. In 1989, Jameson ${ }^{1}$ presented a review on the requirements for the use of numerical techniques to design aircrafts. He pointed out that experimental design is a very expensive tool, where 20000 hours of wind tunnel testing were expended in development of some modern aircrafts like F-111 and Boeing 747. For this reason, the CFD opens new direction to tackle with such engineering problems. In 1994, Jameson ${ }^{3}$ described a method which was used for the calculation of both 2-D and 3-D flows using finite difference scheme, which had been successfully applied to variety of flows over airplane wings. He concluded that near the leading edge of the wing, where the surface curvature was high, the best treatment was the curvilinear coordinate system, in which, the body surface coincided with a coordinate surface. The shock capturing in supersonic flows using CFD technique was carried out and reported by many researchers, e.g. Chen and Dehua ${ }^{4}$ and Persson et al. ${ }^{5}$. Many research results have been reported to solve the supersonic viscous flow field numerically; e.g. Cebeci ${ }^{6}$ who solved the flow around simple axisymetric missiles using Reynolds-average Navier-Stokes equation; DeSpirito et al. ${ }^{7}$ investigated flow around canard controlled missile and lattic fins tail control. Morgenstern ${ }^{8}$ simulated viscous supersonic flow over spiked-nosed body of revolution by solving NavierStokes equations. Solving the supersonic flow assuming its invisid had been proven to produce accurate results and, with suitable grid generation technique, the shock wave could be captured. Wardlaw et al. ${ }^{9}$ solved Euler equations in supersonic speed around missile and predicted the 
aerodynamic parameters and the velocity profile excluding the boundary layer. He used the explicit McCormack's space marching technique. Goonko et al. ${ }^{10}$ presented numerical and analytical prediction of the supersonic invicid steady supersonic flow in corners formed by interaction wedges. They solved 3-D steady Euler equations by space marching approach. Pattman et al. ${ }^{11}$ used finite difference analogue of the full-potential equation to solve the supersonic flow around a missile body. The solution was carried out by NCREL ready code. In their work, grid points around the body surface were automatically clustered near the leading edge. Scalabrin et al. ${ }^{12}$ simulated the supersonic flow around a multistage rocket. The 3-D transonic and supersonic compressible flow was modeled by solving Euler equations and a Taylor-Galerkin finite element method with artificial dissipation was employed. $\mathrm{Wu}$ et al. ${ }^{13}$ presented the results of using arc-length mesh generation and finite volume approach to solve Euler equations. The aim of their work was to improve the liftto-drag ratio of supersonic missiles.

In the present study, the supersonic flow field over seamless missile is simulated by solving Euler equations. The predictor-corrector McCormack's explicit finite difference method is used to predict the aerodynamic properties of 3-D, external compressible, inviscid supersonic flow. The time- marching method is chosen to treat the supersonic seamless missile. The paper discuses, also, the discretization of the resulted set of equations from the mathematical model and how they are converted to a numerical domain to be solved iteratively. The developed technique which is used to produce the grids is presented. An in-house code is developed to generate the grids and to solve the numerical model and perform prediction of the primitive variable covers, velocity, density, temperature, pressure, internal energy and Mach number.

\section{Mathematical Formulation}

The fluid within the present supersonic flow is treated as a non-viscous, non-heat conducting fluid, so it is desirable to be described by Euler equation. This is a valid approximation for flows at high speed (supersonic flow), i.e., at high Reynolds number outside the viscous region developing near the solid surface. The mathematical behavior of the Euler equation in such case is classified as hyperbolic in supersonic flow ${ }^{2}$. The governing equations derived from the conservation principles for an inviscid, non-heat conducting, external, compressible, unsteady, and three-dimensional supersonic flow are expressed in conservation format. By assuming non-viscous fluid, all the shear terms could be omitted from Navier-Stokes equations, getting the conservation of momentum in the flow field as:

$$
\begin{aligned}
& \frac{\partial(\rho u)}{\partial t}+\frac{\partial\left(\rho u^{2}+p\right)}{\partial x}+\frac{\partial(\rho u v)}{\partial y}+\frac{\partial(\rho u w)}{\partial z}=0 \\
& \frac{\partial(\rho v)}{\partial t}+\frac{\partial(\rho u v)}{\partial x}+\frac{\partial\left(\rho v^{2}+p\right)}{\partial y}+\frac{\partial(\rho v w)}{\partial z}=0 \\
& \frac{\partial(\rho w)}{\partial t}+\frac{\partial(\rho u w)}{\partial x}+\frac{\partial(\rho w v)}{\partial y}+\frac{\partial\left(\rho w^{2}+p\right)}{\partial z}=0
\end{aligned}
$$

By neglecting the heat-conduction terms in the general energy equation, the conservation of energy in the flow field becomes:

$$
\begin{gathered}
\frac{\partial\left(\rho E_{t}\right)}{\partial t}+\frac{\partial}{\partial x}\left[\left(\rho E_{t}+p\right) u\right]+\frac{\partial}{\partial y}\left[\left(\rho E_{t}+p\right) v\right] \\
+\frac{\partial}{\partial z}\left[\left(\rho E_{t}+p\right) w\right]=0
\end{gathered}
$$

While the conservation of mass within the flow field, represented by the continuity equation, and could be presented as:

$$
\frac{\partial \rho}{\partial t}+\frac{\partial(\rho u)}{\partial x}+\frac{\partial(\rho v)}{\partial y}+\frac{\partial(\rho w)}{\partial z}=0
$$

The grid points are generated in physical plane and transformed to computational plane before solving governing equations. The solution is obtained by marching from some initial flow field through time until a steady state is obtained. Prior to applying a numerical scheme to solve the set of five equations, it is essential to put them in a vector form as follows:

$$
\frac{\partial Q}{\partial t}+\frac{\partial E}{\partial x}+\frac{\partial F}{\partial y}+\frac{\partial G}{\partial z}=0
$$

where Q, E, F and G are column vectors which are defined as:

$$
Q=\frac{\partial}{\partial t}=\left[\begin{array}{c}
\rho \\
\rho u \\
\rho v \\
\rho E_{t}
\end{array}\right]
$$




$$
\begin{gathered}
E=\frac{\partial}{\partial x}=\left[\begin{array}{c}
\rho u \\
\rho u^{2}+P \\
\rho u v \\
\left(\rho E_{t}+P\right) u
\end{array}\right] ; \\
F=\frac{\partial}{\partial y}=\left[\begin{array}{c}
\rho v \\
\rho u v \\
\rho v^{2}+P \\
\left(\rho E_{t}+P\right) v
\end{array}\right] ; \text { and } \\
G=\frac{\partial}{\partial z}=\left[\begin{array}{c}
\rho w \\
\rho u w \\
\rho w^{2}+P \\
\left(\rho E_{t}+P\right) w
\end{array}\right] .
\end{gathered}
$$

To establish a close domain of solution, the state equation will add a sixth source for interrelating the flow field properties and support the mathematical formational. The approaching air could be characterized mathematically, where, the free stream Mach number is given by:

$$
M_{\infty}=\sqrt{\gamma R T_{\infty}}
$$

And assuming the working fluid, which is air, as a perfect gas, the equation of state reads

$$
\rho_{\infty}=\frac{p_{\infty}}{R T_{\infty}}
$$

While, the total pressure and temperature are given by:

$$
\begin{gathered}
p_{o}=p_{\infty}\left[1+\frac{(\gamma-1)}{2} M^{2}\right]^{\frac{\gamma}{\gamma-1}} \\
T_{o}=T_{\infty}\left[1+\frac{(\gamma-1)}{2} M^{2}\right]
\end{gathered}
$$

Moreover the velocity components are defined by: -

$$
\begin{aligned}
& u_{\infty}=V \cos \alpha=M \cdot a_{\infty} \cos \alpha \\
& v_{\infty}=V \sin \alpha=M \cdot a_{\infty} \sin \alpha
\end{aligned}
$$

Noting that, $\alpha$ is the angle of attack. The properties of air are evaluated at an altitude of, $H=1500 \mathrm{~m}$, using the atmosphere relations ${ }^{14}$ as:

$$
T_{\infty}=288.16-0.0065 H
$$

and,

$$
P_{\infty}=101325\left(\frac{T_{\infty}}{288.16}\right)^{\left(\frac{0.03415 H}{288.16-T_{\infty}}\right)}
$$

Providing that $\mathrm{T}_{\infty}=278.4 \mathrm{~K}$, and the pressure, $\mathrm{p}_{\infty}=84,643.0 \mathrm{~Pa}$. At Mach number equals 1.5, the free stream velocity becomes $501.6 \mathrm{~m} / \mathrm{s}$.

\section{Three Dimensional Mesh Generation}

In this work, the algebraic grid generation method is used to produce the computational mesh. This method generates grid points in space by means of interpolations based on given boundary data. Because of the non-uniform shape of seamless missile, a 'body-fitted coordinate system' is used. This grid generation method enables transformation of governing equations from a Cartesian $\operatorname{system}(x, y, z)$ to a general curvilinear system $(\xi, \eta, \zeta)$ and each vector is transformed from the physical space domain to the computational domain ${ }^{15}$.

\subsection{Computational Domain}

3-D domain is chosen over the supersonic seamless missile, which has diameter of $306 \mathrm{~mm}$, and geometries shown in Figure 1.

The domain is discretized to $(21 \cdot 32 \cdot 21)$ elements in $\mathrm{x}, \mathrm{y}$, and $\mathrm{z}$ directions respectively. The computational domain is solved within boundaries of $\mathrm{im}=21, \mathrm{jm}=16$ and $\mathrm{km}=21$, where:

$\mathrm{im}=$ maximum number of grid nodes in $\mathrm{x}$-direction. $\mathrm{jm}=$ maximum number of grid nodes in y-direction. $\mathrm{km}=$ maximum number of grid nodes in $\mathrm{z}$-direction.

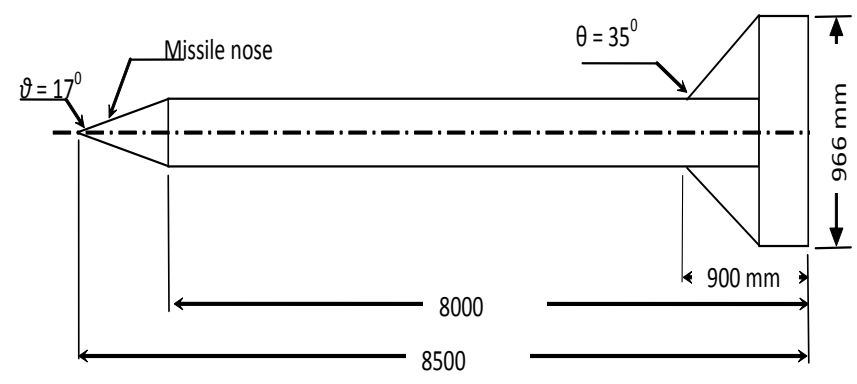

Figure 1. Geometries of the seamless missile. 


\subsection{Axis Transformation}

The transformation of any partial differential term from physical domain, described in $x, y$, and $z$ coordinate system, to computational domain characterized by $\xi$, $\eta$, and $\zeta$ could be defined as following:

$$
\begin{aligned}
& \xi=\xi(x, y, z) \\
& \eta=\eta(x, y, z) \\
& \zeta=\zeta(x, y, z)
\end{aligned}
$$

The transformation procedure is very lengthy and complex. For more details, it is recommended to refer to ${ }^{16}$. By defining the Jacobian transformation, which represents the ratio of the volume of any element in the physical domain to its volume in the computational domain, as:

$$
J=\frac{1}{x_{\xi}\left[y_{\eta} z_{\zeta}-z_{\eta} y_{\zeta}\right]-x_{\eta}\left[y_{\xi} z_{\zeta}-z_{\xi} y_{\zeta}\right]+x_{\zeta}\left[y_{\xi} z_{\eta}-z_{\xi} y_{\eta}\right]}
$$

Then, matrices of space transformation are representing the ratio of arc length in the computational space to that of the physical space, and defined as follows:

$$
\begin{aligned}
& \xi_{x}=J {\left[y_{\eta} z_{\zeta}-y_{\zeta} z_{\eta}\right] } \\
& \xi_{y}=-J\left[x_{\eta} z_{\zeta}-x_{\zeta} z_{\eta}\right] \\
& \xi_{z}=J\left[x_{\eta} y_{\zeta}-x_{\zeta} y_{\eta}\right] \\
& \eta_{x}=-J\left[y_{\xi} z_{\zeta}-y_{\zeta} z_{\xi}\right] \\
& \eta_{y}=J\left[x_{\xi} z_{\zeta}-x_{\zeta} z_{\xi}\right] \\
& \eta_{z}=-J\left[x_{\xi} y_{\zeta}-x_{\zeta} y_{\xi}\right]
\end{aligned}
$$

and

The resulted 3 dimensional discretized domain is shown in Figure 2. Half of the flow field is considered for simulation by utilizing the symmetry up and down the missile.

\section{Numerical Scheme}

A finite difference with axis transformation technique is adopted to solve the present problem, numerically. Explicit

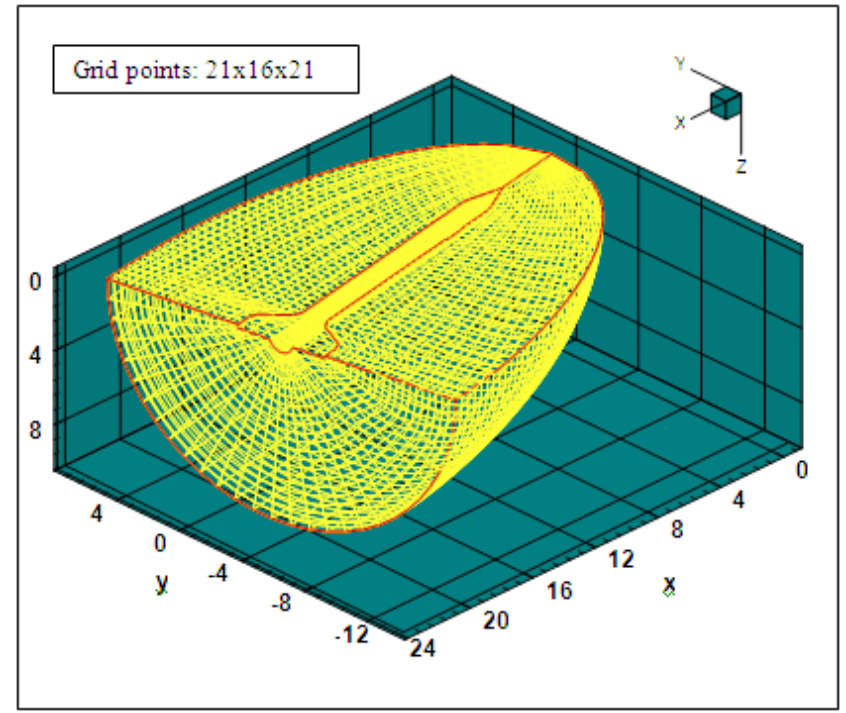

Figure 2. Algebraic grid generation over a 3-D supersonic seamless missile with mesh points $(21 \cdot 16 \cdot 21)$ in $x, y$ and $z$ Cartesian coordinates considering half of the symmetrical domain.

time-dependent solution of the 3-D Euler equations has been performed using MacCormack's predictor-corrector finite difference technique, which is a second-order accurate in both space and time. This method is very effective finite difference technique for viscous and inviscid supersonic flows, especially for unsteady flow shock capturing ${ }^{16}$. The terms $x_{\zeta}, x_{\eta}, y_{\zeta}, \ldots$ etc., are computed numerically using forward approximations; for a example:

$$
y_{\eta}=\frac{\partial y}{\partial \eta}=\frac{-3 y_{i, j, k}+4 y_{i, j+1, k}-y_{i, j+2, k}}{2 \Delta \eta}
$$

The time step employed is designed so that it shouldn't exceed the maximum step size permitted with maintaining the stability of iteration solution. In this study, the inviscid Courant Fridrich Lewys (CFL) stability condition is adopted, which is given by ${ }^{17}$ :

$\left.\Delta t\right|_{C F L} \leq\left[\frac{|u|}{\Delta x}+\frac{|v|}{\Delta y}+\frac{|w|}{\Delta z}+a \times\left[\frac{1}{(\Delta x)^{2}}+\frac{1}{(\Delta y)^{2}}+\frac{1}{(\Delta z)^{2}}\right]^{\frac{1}{2}}\right]^{-1}$

Changing of primitive variables to fluxes is performed to compute the values of flux vectors for all grid points at time step (n).A forward predictor version of MacCormack's which is given by ${ }^{16}$ : 


$$
\begin{aligned}
\bar{Q}_{i, j, k}^{n+1}= & \bar{Q}_{i, j, k}^{n}-\frac{\Delta t}{\Delta \xi}\left[\bar{E}_{i+1, j, k}^{n+1}-\bar{E}_{i, j, k}^{n+1}\right]-\frac{\Delta t}{\Delta \eta}\left[\bar{F}_{i, j+1, k}^{n+1}-\bar{F}_{i, j, k}^{n+1}\right] \\
& -\frac{\Delta t}{\Delta \zeta}\left[G_{i, j, k+1}^{n+1}-G_{i, j, k}^{n+1}\right]
\end{aligned}
$$

It is used inside the domain up to nodes just before the last boundaries. The flow parameters, such as $u, v, w, M, T$, $e, \rho$ and $p$ are computed at the plane $(\mathrm{k}=1)$. Then they are computed in the domain except at the surface. The superscript, $n$, in equation (16) is the time level ( $t$ ), and $n+1$ is the time level $(t+d t)$. In order to stabilize and improve the accuracy of the procedure, an artificial viscosity term is imposed to the predictor step. For that, a fourth-order artificial viscosity term, $S Q_{i, j, k}^{n+1}$ in the form suggested by ${ }^{18}$ is added as follows:

$$
\begin{aligned}
S Q_{i, j, k}^{n+1}= & C_{\xi} \frac{\left|P_{i-1, j, k}^{n}-2 P_{i, j, k}^{n}+P_{i-1, j, k}^{n}\right|}{P_{i-1, j, k}^{n}+2 P_{i, j, k}^{n}+P_{i-1, j, k}^{n}} \\
& \left.\left.\left.\times[\bar{Q})_{i-1, j, k}^{n}-2 \bar{Q}\right)_{i, j, k}^{n}+\bar{Q}\right)_{i+1, j, k}^{n}\right] \\
& +C_{\eta} \frac{\left|P_{i, j-1, k}^{n}-2 P_{i, j, k}^{n}+P_{i, j+1, k}^{n}\right|}{P_{i, j-1, k}^{n}+2 P_{i, j, k}^{n}+P_{i, j+1, k}^{n}} \\
& \left.\left.\left.\times[\bar{Q})_{i, j-1, k}^{n}-2 \bar{Q}\right)_{i, j, k}^{n}+\bar{Q}\right)_{i, j+1, k}^{n}\right] \\
+ & C_{\zeta} \frac{\left|P_{i, j, k-1}^{n}-2 P_{i, j, k}^{n}+P_{i, j, k+1}^{n}\right|}{P_{i, j, k-1}^{n}+2 P_{i, j, k}^{n}+P_{i, j, k+1}^{n}} \\
& \left.\left.\left.\times[\bar{Q})_{i, j, k-1}^{n}-2 \bar{Q}\right)_{i, j, k}^{n}+\bar{Q}\right)_{i, j, k+1}^{n}\right]
\end{aligned}
$$

This in fact, an explicit artificial dissipation which is important since the flow field is at high Reynolds number, and compressible nature. The main advantage of artificial dissipation is to provide some mathematical dissipation analogous to the real viscous effects within the shock wave region. At this step, the contravariant velocity components are defined and computed by:

$$
\begin{aligned}
& \mathrm{U}=\xi_{x} u+\xi_{y} v+\xi_{z} \omega \\
& \mathrm{V}=\eta_{x} u+\eta_{y} v+\eta_{z} w \\
& \mathrm{~W}=\zeta_{x} u+\zeta_{y} v+\zeta_{z} w
\end{aligned}
$$

The contravarient velocities ( $\mathrm{U}, \mathrm{V}$ and $\mathrm{W}$ ) represent velocity components which are perpendicular to planes of constant $\xi, \eta$, and $\zeta$. Then, a corrector step is computed, where the values of fluxes $E, F$, and $G$ are computed at each grid point in the intermediate level $(n+1)$ depending on the values of primitive variables from previous step.
Moreover, a backward corrector version of MacCormack's method which is given by ${ }^{16}$ is then calculated and applied as:

$$
Q_{i, j, k}^{n+1}=(1 / 2)^{\star}\left\{\begin{array}{l}
Q_{i, j, k}^{n}+\bar{Q}_{i, j, k}^{n+1}-\frac{\Delta t}{\Delta \xi}\left[\bar{E}_{i, j, k}^{n+1}-\bar{E}_{i, j-1, k}^{n+1}\right] \\
-\frac{\Delta t}{\Delta \eta}\left[\bar{F}_{i, j, k}^{n+1}-\bar{F}_{i, j-1, k}^{n+1}\right]-\frac{\Delta t}{\Delta \zeta}\left[G_{i, j, k}^{n+1}-G_{i, j-1, k}^{n+1}\right]
\end{array}\right\}
$$

This corrector formula is used inside the domain and, also a fourth-order artificial viscosity term at corrector step is added to Equation (19). This expression is given by $^{18}$ :

$$
\begin{aligned}
\left.S Q^{n+1}\right)_{i, j, k}= & C_{\xi} \frac{\left|P_{i+1, j, k}^{n+1}-2 P_{i, j, k}^{n+1}+P_{i-1, j, k}^{n+1}\right|}{\left[P_{i+1, j, k}^{n+1}-2 P_{i, j, k}^{n+1}+P_{i-1, j, k}^{n+1}\right]} \\
& \left.\left.\left.*\left[\bar{Q}_{1}\right)_{i-1, j, k}^{n+1}-2 \bar{Q}_{1}\right)_{i, j, k}^{n+1}+\bar{Q}_{1}\right)_{i+1, j, k}^{n+1}\right] \\
& +C_{\eta} \frac{\left|P_{i, j+1, k}^{n+1}-2 P_{i, j, k}^{n+1}+P_{i, j-1, k}^{n+1}\right|}{\left[P_{i, j+1, k}^{n+1}-2 P_{i, j, k}^{n+1}+P_{i, j-1, k}^{n+1}\right]} \\
& \left.\left.\left.*\left[\bar{Q}_{1}\right)_{i, j-1, k}^{n+1}-2 \bar{Q}_{1}\right)_{i, j, k}^{n+1}+\bar{Q}_{1}\right)_{i, j+1, k}^{n+1}\right] \\
& +C_{\zeta} \frac{\left|P_{i, j, k+1}^{n+1}-2 P_{i, j, k}^{n+1}+P_{i, j, k-1}^{n+1}\right|}{\left[P_{i, j, k+1}^{n+1}-2 P_{i, j, k}^{n+1}+P_{i, j, k-1}^{n+1}\right]} \\
& \left.\left.\left.*\left[\bar{Q}_{1}\right)_{i, j, k-1}^{n+1}-2 \bar{Q}_{1}\right)_{i, j, k}^{n+1}+\bar{Q}_{1}\right)_{i, j, k+1}^{n+1}\right]
\end{aligned}
$$

Once the corrector step is completed, the code recomputed the parameters $(\mathrm{u}, \mathrm{v}, \mathrm{w}, \mathrm{T}$, e and $\mathrm{M})$.After that, the following convergence criterion ${ }^{19}$ is utilized at every point in the flow field from one time step to the next:

$$
\text { error }=\frac{\rho_{\text {old }}-\rho_{\text {new }}}{\rho_{\text {old }}} \leq 1^{*} 10^{-8}
$$

\section{Results and Discussion}

In order to verify the results of the present work, a familiar case is selected for checking the efficiency of the present code. For comparison, the current program which is used to capture the shock wave and aerodynamic flow field parameters around a seamless missile is used to capture the shock wave and Mach number contours (as a selected case) for a double wedge with a wedge angle $=15$ degree and a free stream Mach number equals to 2.5. The program of the present study is capable to capture the shock 
wave and the flow field simulation as demonstrated in Figure 3.

A computer code is established to conduct the iterative solution procedure of the set of governing equations presented in the mathematical model and explained in the numerical solution scheme. The explicit technique has required about (4000) time steps to achieve the converged solution (steady state solution).In the present work, the artificial viscosity is taken as (0.5).

The results demonstrate that the numerical scheme which is used in the present study successful to capture the shock wave, which can be noticed clearly ahead of the nose of the seamless missile in Figure 4. The shock wave is observed at a head of missile' nose where the incoming flow is supersonic, and the flow nature behind the shock wave near the leading missile' nose area becomes subsonic flow. The Mach number at the outer domain of the flow field represents the free stream value which in this case equals to 1.5. This figure exhibits also, that in order to capture the flow field around a missile precisely; a more grid points are required near the body surface. The captured shock wave is created due to pressure differences in the flow field regions. From this figure, the flow pattern near the leading edge of supersonic seamless missile, where the incoming supersonic flow undergoing a sudden change in flow direction, is resulting in a continuous compression wave. The angle of the shock wave depends on the nose shape and free stream Mach number. The

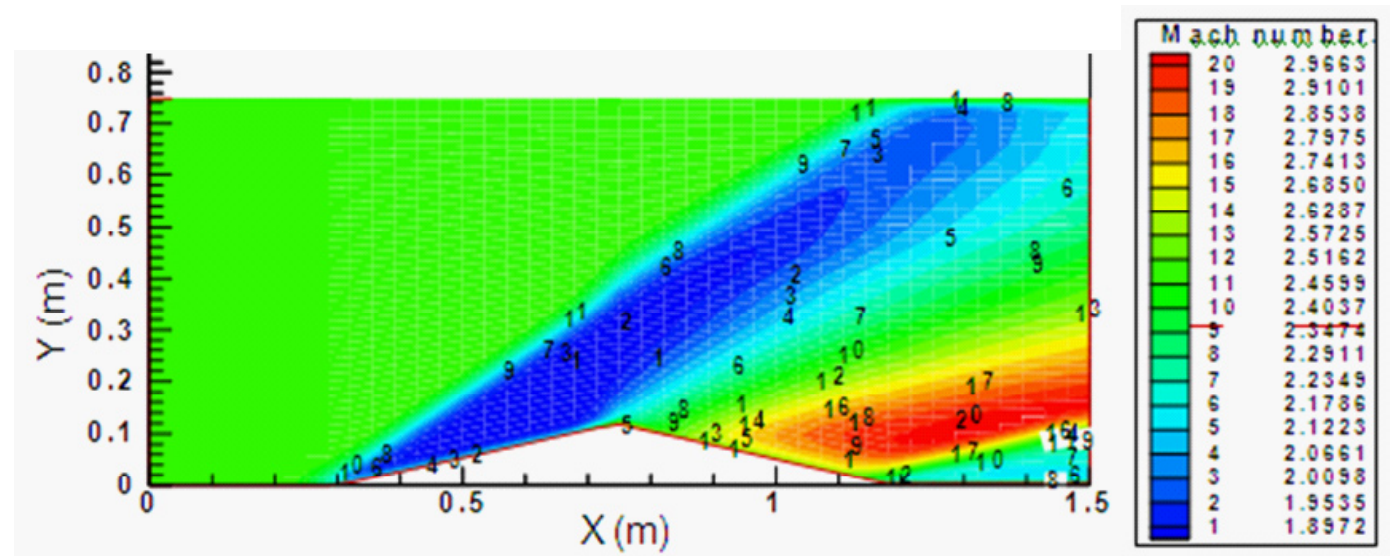

Figure 3. Mach number contours over a double wedge used for comparison with wedge angle $=15$ degree at free stream Mach number $=2.5$. mesh $63 \cdot 53$.

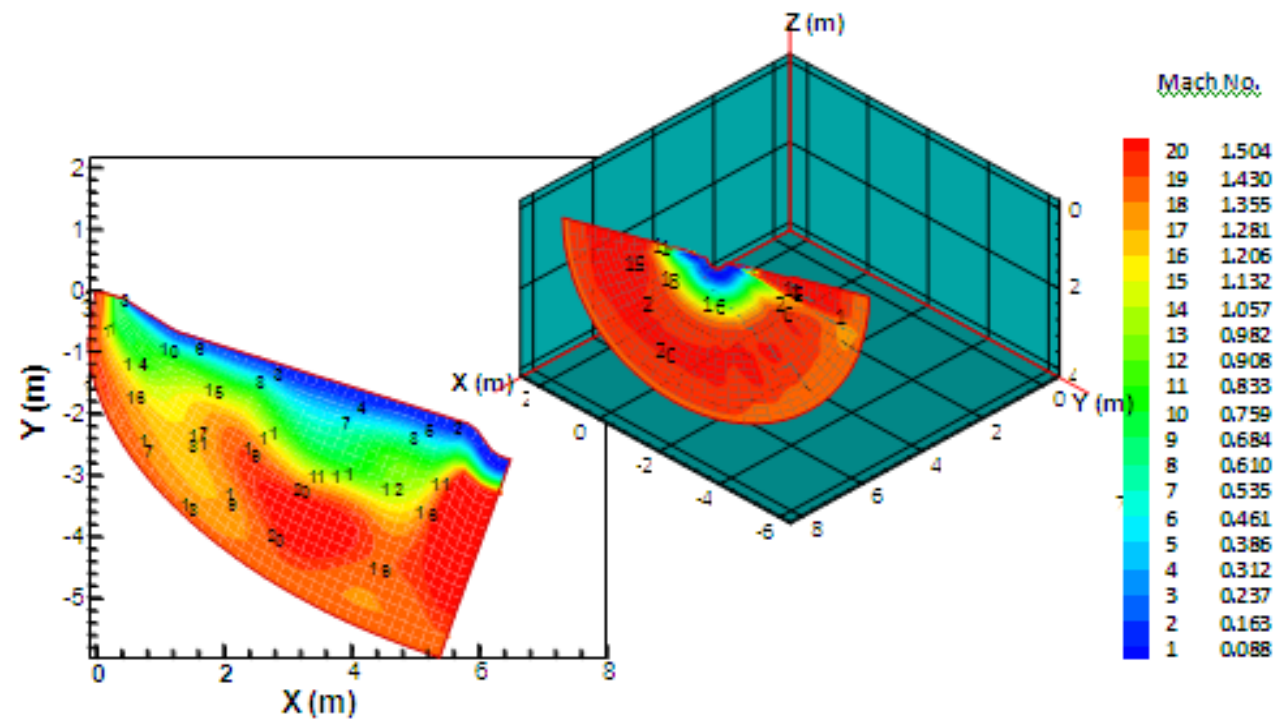

Figure 4. The Mach number contours along the missile and in $\mathrm{z}-\mathrm{y}$ plane at the end of the seamless missile for free stream Mach number $=1.5$. 
shock is observed to be detached from missile nose angle and flow behind the shock near the trailing edge of missile area becomes subsonic. Also, it is very important to refer that the clustering process is very necessary near the leading edge of supersonic missile in order to capture all the expected shock waves. This prediction gives a good agreement with the experimental results dealing with the same problem as indicated in ${ }^{20}$. It is very useful to note that the predicted shock wave by the numerical solution of the present work decelerates the flow speed from supersonic speed up stream of the shock wave to subsonic flow downstream of the shock wave.

In Figure 5, a temperature contours for supersonic seamless missile for free stream Mach number $=1.5$ is explained. This figure indicates that the temperature distribution occurs at the region between the missile surface and the shock wave. Also, the temperature increases at the leading edge of the missile due to shock wave strength and is decreased gradually toward its free stream value. The rise in the value of temperature distribution at the stagnation point is due to flow nature change from supersonic a head of the shock wave to subsonic downstream of the shock wave. This change will reduce the kinetic energy and at the same time, this reduction gives an increase in internal energy and as a result increases the temperature. It is very important to indicate that this high temperature affects on the surface and the structure of the missiles and can cause the melting of the edges and corners of the missile, when the temperature exceeds the melting temperature point of the seamless missile body material. The higher temperature can be noticed at the edge of three-dimensional supersonic seamless missile, due to shock wave effect.

Figure 6 show a pressure contours over the seamless missile. The pressure values increase a head of shock wave and then decrease toward its free stream value away from the shock wave. This is agreeing with ${ }^{21}$ and ${ }^{22}$ also, the pressure values are increased dramatically near the leading edge of supersonic seamless missile due to shock wave effects. A high pressure is detected near the nose of the missile. This is again due to strong shock wave which is predicted at the leading edge of the missile. The reason of this high value of the pressure can be go back due to large amount of friction only, since the current numerical scheme neglects the effect of hydrodynamic and thermal boundary layer which can be predicted by considering viscous flow around the missile rather than the inviscid flow which is simulated in the present work.

Figure 7 shows a density contours over a threedimensional supersonic seamless missile for free stream Mach number $=1.5$. From this figure, the density values increase due to increase of the pressure and this is due to increase in shock wave strength. The high pressure values cause an increase in density values due to the same reason explained above and this behavior can be noticed in Figure 7. Normally, the increase in pressure causes a corresponding increase in density due to direct proportionality between them through the state equation. Moreover, it is found in both Figure 6 and 7 that the pressure and density values after the shock wave are greater

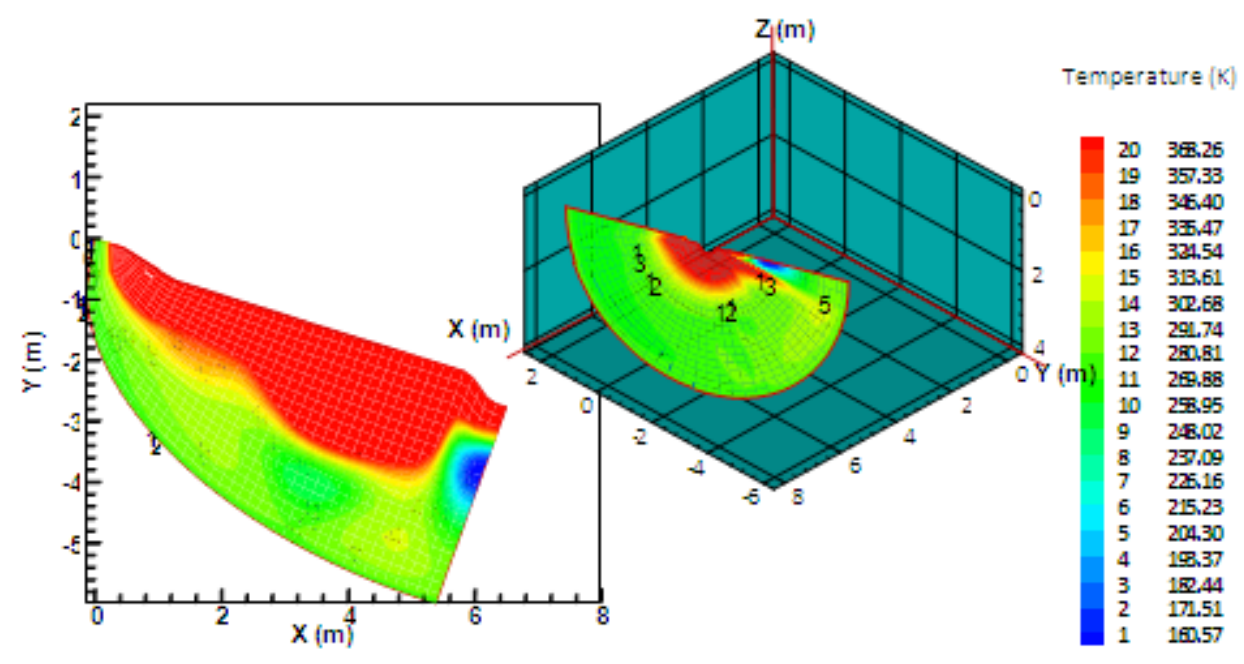

Figure 5. The temperature distribution along the missile, and in z-y plane at the end of the seamless missile for free stream Mach number $=1.5$. 


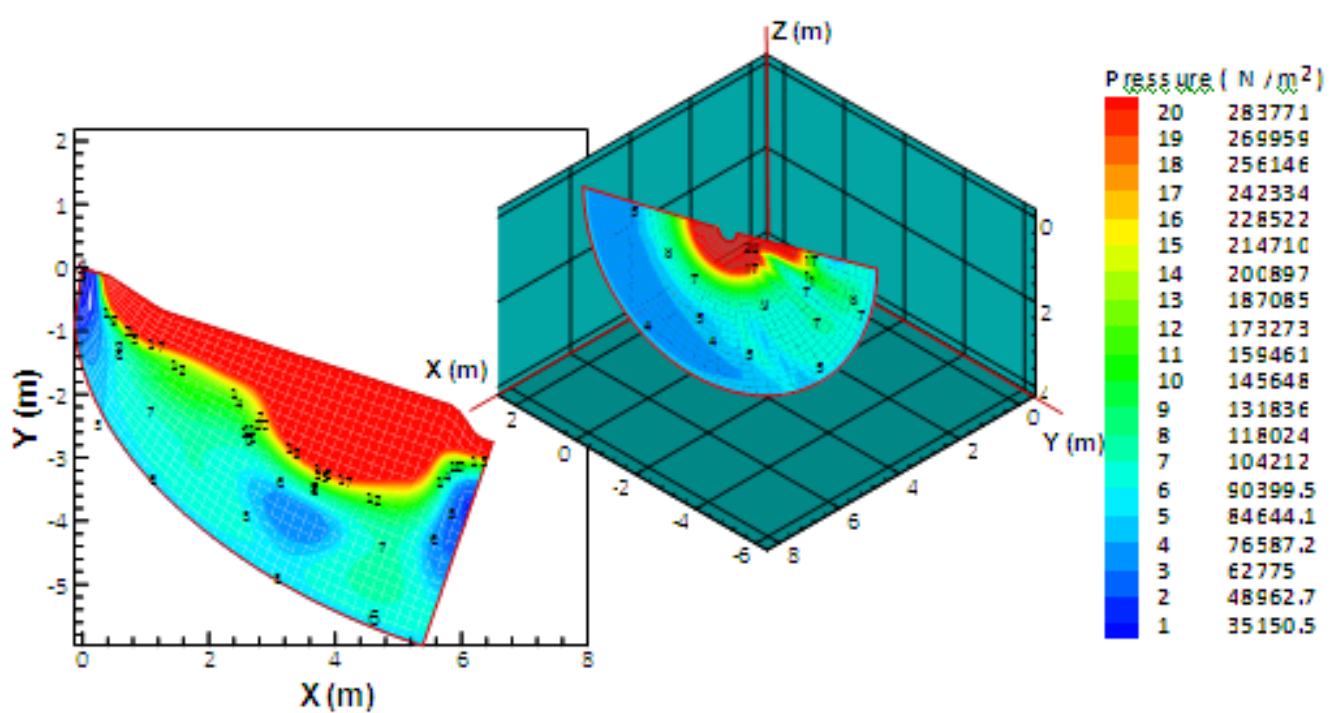

Figure 6. The pressure distribution along the missile, and at $\mathrm{z}-\mathrm{y}$ plane at the end of the seamless missile for free stream Mach number $=1.5$.

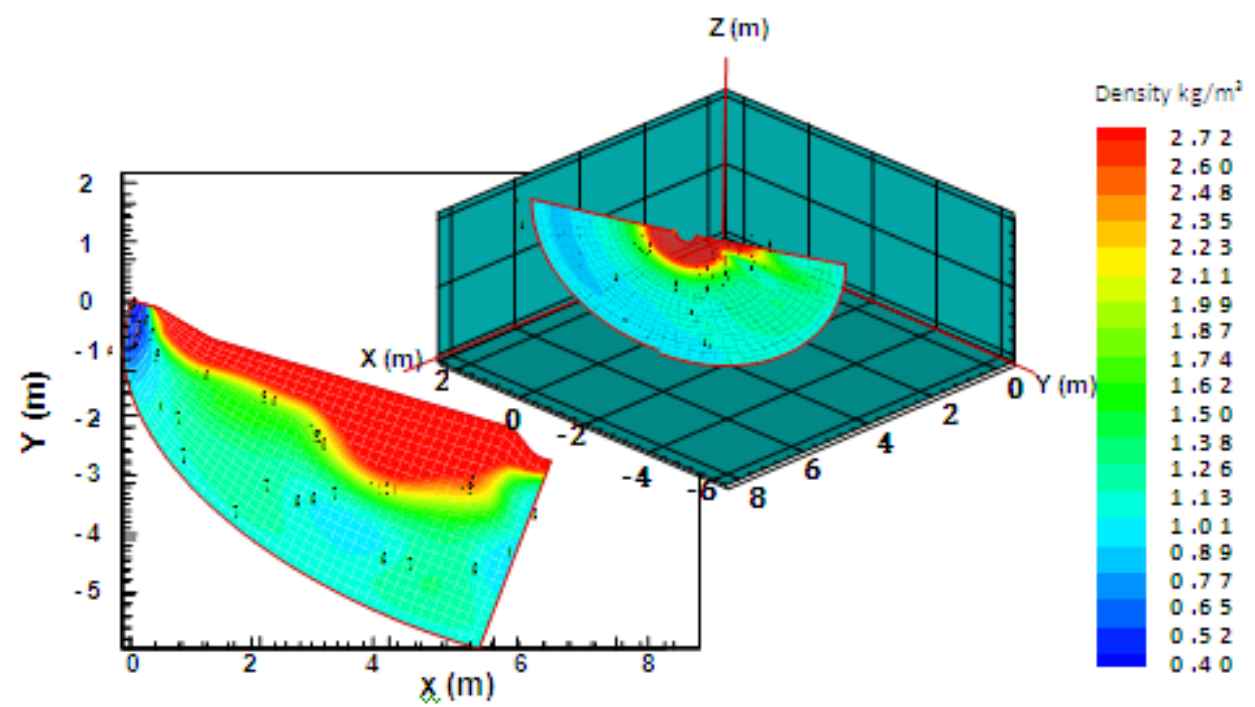

Figure 7. The density distribution along the missile, and at $\mathrm{z}-\mathrm{y}$ plane at the end of the seamless missile for free stream Mach number $=1.5$.

than the corresponding values before the shock wave. This means that the shock wave decelerates the flow speed from supersonic to subsonic speed.

\section{Conclusion}

An in house computer code to simulate and predict the flow field surrounding seamless missile has been implemented and validated. Finite difference CFD methods are adopted to solve the governing equations of supersonic, inviscid, compressible, and three-dimensional flow over the missile body with no canard. The following conclusions can be drawn from the results of the present work:

The shock wave is observed to be detached from the seamless missile nose angle and flow behind the shock near the trailing edge becomes subsonic. The captured shock wave causes a high friction near a nose of the seamless missile. This high friction creates a high temperature, pressure, density and internal energy. The temperature gradient is high at the leading edge of the seamless mis- 
sile due to shock wave strength and is decreased gradually toward its free stream value. A high temperature field may be noticed near the missile nose and wings regions. The hydrodynamic properties of the flow field around a seamless missile such as pressure, temperature and density are increased due to shock wave effect. Also, they vary from higher values near the head of the missile to their free stream values near the outer boundary of the domain. The pressure and density values after the shock wave are greater than the corresponding values before the shock wave. This indicates that the shock wave decelerates the flow speed from supersonic to subsonic speed.

A more mesh points are required near the surface of the supersonic seamless missile. Moreover, the clustering process is found to be very significant adjacent the leading edge of supersonic missile in order to capture all the expected shock waves.

As continuation to the analysis of the flow surrounding the seamless missiles, it is recommended to run the code at different Mach numbers to investigate its effect on the flow field structure. Also, it is worth to compare between the cases of seamless and canard missiles. This will improve the understanding of the canards effect on the flow field surrounding the missiles.

\section{Acknowledgement}

The authors acknowledge Universiti Teknologi PETRONAS for the technical and financial support to publish the research results.

\section{References}

1. Jameson A. Computational Aerodynamics for Aircraft Design. Science Journal. 1989; 245:361-71.

2. Weatherill NP, Forsey CR. Grid Generation and Flow Calculations for Aircraft Geometries. J Aircraft. 1985; 22:855-60.

3. Jameson A. Iterative Solution of Sonic Flows Over Airfoils and Wings, Including Flows at Mach 1.0. Comm Pure Appl Math. 1994; 27:283-309.

4. Chen A, Dehua J. Shock-Capturing and Related Numerical Methods in Computational Fluid Dynamics. Communications of Mathematics and Physics. 1996; 101-10.

5. Persson, M, Rizzi A, Pettersson K. Aerodynamics of Hypervelocity Missiles. Department of Aeronautics, KTH: 1998. 5 p. Report 98-40
6. Cebeci T. Three-Dimensional Boundary Layers on Missiles. NASA Technical Report. 2000. 55 p.

7. DeSpirito J, Vaughn ME, Washington W. CFD Investigation of Canard-Controlled Missile with Planar and Grid Fins in Supersonic Flow. AIAA Atmospheric Flight Mechanics Conference and Exhibit; 2002; California, U.S.A.

8. Morgenstern JA. Three-Dimensional Supersonic Flow Over a Spike - Nosed Body of Revolution. J Braz Soc Mech Sci. 2002 Nov; 24(4):271-7.

9. Wardlaw A, Solomon J, Baltakis F. Euler Space Marching Methods Applied to Missiles. NASA Technical Report. 2000. 53 p.

10. Goonko YP, Kudryavtsev AN, Rakhimov RD. Supersonic Inviscid Flows with Three-Dimensional Interaction of Shock Waves in Corners Formed by Intersection Wedges. European Congress on Computational Methods in Applied Science and Engineering. 2002; 79-85.

11. Pittman JL, Miller DS, Siclari MJ. Supersonic Full-Potential Method Applied to Missile Bodies. NASA Technical Report. NASA Langley Research Center: 2002. 36 p.

12. Scalabrin LC, Azevedo JL, Teixeira PR, Awruch AM. ThreeDimensional Flow Simulations with the Finite Element Technique Over a Multi-Stage Rocket. J Braz Soc Mech Sci Eng. 2004; 26(2):107-16.

13. Wu P, Yong-Gang M, Chun C. The Research Analysis of Aerodynamic Numerical Simulation of Grid Fin. Journal of Zhejiang University Science. 2005; 6A(7):741-6.

14. Bertin JJ, Smith ML. Aerodynamics for Engineers. 3rd ed. London: Prentice Hall International Editions; 1998.

15. Fox M. Supersonic Aerodynamic Characteristic of an Advanced F-16 Derivative Aircraft Configuration. NASA; 1993. 22 p. Technical Paper 93-3355

16. Hoffmann AK. Computational Fluid Dynamics for Engineering. University of Texas; 1989.

17. AL-Dulaimy FM. Numerical Prediction of Supersonic Inviscid and Viscous Flow over Arbitrary Configurations [PhD thesis]. Baghdad: University of Technology; 2002.

18. Anderson JD. Computational Fluid Dynamics: The Basic withApplications.U.S.A.:McGraw-HillBookCompany;1995.

19. Wylie CR. Advanced Engineering Mathematics. International Student ed. U.S.A.: MacGraw -Hill Book Company; 1966.

20. Marsden O, Bogey C, Bailly C. Higher-Order Curvilinear Simulations of Flows around Non-Cartesian Bodies. AIAA paper 2004-2813. 2004:1-15.

21. Orensen N. 3-D Background Aerodynamics Using CFD. Roskilde, Denmark: Riso National Laboratory Publications; 2002.

22. Dietz W, Wang L, Wenren Y, Caradonna F, Steinhoff J. The Development of a CFD-Based Model of Dynamic Stall. 60th Annual Forum; 2004; Baltimore U.S.A. AHS; 2004. p. 1-17. 